\title{
THE RELATIONSHIP BETWEEN TOTAL GAS CONTENT OF POLAR ICE, ATMOSPHERIC PRESSURE AND SURFACE ELEVATION
}

\author{
(Abstract) \\ by \\ P. Martinerie, D. Raynaud and D. Mazaudier \\ (Laboratoire de Glaciologie et Géophysique de l'Environnement du C.N.R.S., \\ B.P. 96, 38402 St Martin d'Hères Cédex, France) \\ A.J. Gow \\ (Snow and Ice Branch, U.S. Army CRREL, 72 Lyme Road, Hanover, NH 03755-1290, U.S.A.) \\ G. Holdsworth
}

(Environment Canada, Surface Water Division, National Hydrology Research Institute, 11 Innovation Boulevard, Saskatoon, Saskatchewan S7N 3H5, Canada)

V.Ya. Lipenkov

(Arctic and Antarctic Research Institute, Ul. Beringa 38, 199226 Leningrad, U.S.S.R.)

and

N.W. Young

(Australian Antarctic Division, Channel Highway, Kingston, Tasmania 7050, Australia)

\section{ABSTRACT}

The air found in polar ice becomes isolated from the atmosphere during the transformation of snow into ice. The total gas content, $V$, measured on selected ice samples depends theoretically on the atmospheric pressure and temperature prevailing at the ice-formation site and also on the volume, $V c$, of the firn pores at the time they become isolated from the atmosphere in newly formed ice.

In spite of scattered individual values, a clear decrease in mean $V$-results has previously been observed for increasing elevations at the ice-formation site. Assuming that the pores close off at atmospheric pressure, this decrease primarily reflects the pressure-elevation gradient and typically only about $10 \%$ of the decrease can be attributed to the temperature-elevation gradient or changes in $V c$.

Measurements of the total gas content of Antarctic ice cores are potentially the most direct and sensitive indicator of the elevation at which the ice was formed and provide a unique tool for reconstructing the past elevation of the ice-sheet surface. Nevertheless, to make such a reconstruction with confidence, we need first to evaluate the individual contributions of the different parameters which should influence $V c$. Along this line, a stage was reached with the semi-empirical observation of a quasi-linear correlation between mean $V c$ and temperature, although the data points on which the correlation is based are relatively scarce. Furthermore, confidence in the paleo-interpretation of $V$-measurements is dependent also on an understanding of the scatter in individual $V$-values generally observed for a given ice-core section.

New measurements of $V$ are presented, which permit a better and more detailed description to be made of the changes in $V$ with atmospheric pressure and elevation under approximately present-day conditions. As a first step, scattering in the values of individual $V$-measurements of a single core section is investigated. The experimental method used for this work is basically the same as the one used for establishing the relationship between $V$ and elevation, as given by Raynaud and Lebel (1979). Generally measurements performed on the same horizontal layer (about $3 \mathrm{~cm}$ thick) reproduce the same result within the experimental uncertainty (which is $\pm 1 \%$ ). On the other hand, measurements performed on adjacent horizontal layers may show $V$-differences as high as $20-25 \%$. This confirms that the variability of the $V$ measurements that occurs over short intervals in the core is not due to experimental uncertainties and should mainly reflect changes in $V c$.

The main reason for making this new set of measurements was to increase significantly the number of sites investigated in the study of the relationship between $V$ and elevation. The work of Raynaud and Lebel (1979) was based on six sites. The results for 14 sites (in Antarctica, Greenland, and on Mount Logan in Canada) are now available, with elevations ranging from about 340 to $5300 \mathrm{~m}$ and temperatures in the range $-12.4^{\circ}$ to $-55.4^{\circ} \mathrm{C}$. The results confirm a general decrease in $V c$ with temperature. But at least one site shows mean $V$-values which are clearly incompatible with this general trend.

Finally, before proceeding further with studies of the distribution of $V$ versus site parameters, we need to correct our $V$-measurements for the loss of gas which occurs when cutting the ice samples (and consequently opening air bubbles at the sample surface). We have developed, for this purpose, a technique which calculates random distributions of bubbles in the samples and counts the cut bubbles. This calculation depends on bubble shape and size as well as on sample volume and $V$. In the case of the samples used in the present study, between 3 and $10 \%$ of gas is typically lost during cutting. Bubble-size measurements have yet to be made before an accurate correction can be applied to the measurements presented here.

\section{REFERENCE}

Raynaud, D., and B. Lebel. 1979. Total gas content and surface elevation of polar ice sheets. Nature, 281(5729), 289-291. 